\title{
Intratumoral pseudoaneurysm within a liver metastasis of gastric cancer: a case report
}

Hiroki Ohara', Yuji Ishibashi" ${ }^{*}$, Shuntaro Yoshimura', Ryoto Yamazaki², Fumihiko Hatao', Takeshi Koshiishi ${ }^{3}$, Yasuhiro Morita ${ }^{1}$ and Kazuhiro Imamura'

\begin{abstract}
Background: Intrahepatic artery pseudoaneurysms are mostly iatrogenic and result from hepatobiliary interventions. The incidence of intrahepatic artery pseudoaneurysms within liver tumors without prior intervention is extremely rare. We presented herein the first report of a case of an intratumoral pseudoaneurysm within a liver metastasis of gastric cancer without any prior intervention during chemotherapy.

Case presentation: A 59-year-old male patient underwent a distal gastrectomy and D2 lymph node dissection for gastric cancer. He was treated in the emergency room for right abdominal pain following the 4th cycle of nivolumab administration as second-line chemotherapy after adjuvant chemotherapy with S-1 and first-line chemotherapy for a liver metastasis of gastric cancer with ramucirumab plus paclitaxel. CT showed a 72-mm metastatic liver tumor containing a 9-mm pseudoaneurysm and fluid collection around the hepatic edge. Intrahepatic artery pseudoaneurysm within the metastatic liver tumor was diagnosed, with the surrounding fluid indicating potential, active bleeding. An emergency angiography confirmed the presence of a pseudoaneurysm in the intrahepatic artery, which was embolized using microcoils. The contributory causes of the intratumoral pseudoaneurysm were assumed to be the following: (1) tumor necrosis leading to encasement, erosion of the vessel wall, and subsequent arterial wall weakening; and (2) inhibition of vascular endothelial growth by ramucirumab resulting in a vessel wall breach and pseudoaneurysm formation.
\end{abstract}

Conclusion: It is necessary to recognize that pseudoaneurysms can arise within a metastatic liver tumor during chemotherapy.

Keywords: Pseudoaneurysm, Hepatic artery, Gastric cancer, Metastatic liver tumor

\section{Introduction}

Pseudoaneurysms result from the partial to complete disruption of the vascular wall and lead to hemorrhaging contained by the adventitia of the vessel wall or the perivascular soft tissues [1]. Intrahepatic artery pseudoaneurysms are rare and mostly iatrogenic, resulting from hepatobiliary interventions. The incidence of pseudoaneurysms within a liver tumor without prior intervention is extremely rare. We presented herein the first report of a case of intratumoral pseudoaneurysm (ITPA) within a liver metastasis of gastric cancer during chemotherapy without any prior intervention.

\footnotetext{
* Correspondence: ishibashi_y@live.jp

'Department of Surgery, Tokyo Metropolitan Tama Medical Center, 2-8-29

Musashidai, Fuchu-shi, Tokyo 183-8524, Japan

Full list of author information is available at the end of the article
}

\section{Case report}

A 59-year-old male patient underwent a distal gastrectomy and D2 lymph node dissection for gastric cancer. The final pathological stage was T4aN3aM0 Stage IIIC according to the Japanese Gastric Cancer Association Classification, 14th Edition [2]. Postoperative adjuvant chemotherapy with S-1 (100 mg/day p.o., twice daily for 28 consecutive days) was administered for 1 year in accordance with treatment guidelines. One and a half years after surgery, computed tomography (CT) revealed a liver metastasis in hepatic segment VI. The recurrence was detected within 6 months after adjuvant chemotherapy with S-1, and combination therapy with ramucirumab (RAM: $8 \mathrm{mg} / \mathrm{kg}$ on days 1 and 15 ) and paclitaxel (PTX: $80 \mathrm{mg} / \mathrm{m} 2$ on days 1,8 , and 15 ) was administered as first-line chemotherapy, with the regimen repeated at 28-day intervals. After eight cycles with RAM plus PTX, 
follow-up CT revealed a new liver metastasis in segments VI and VIII. Nivolumab (3 mg/kg, biweekly) was administered as second-line treatment. Five days after the 4th cycle of nivolumab injections, the patient visited our emergency room due to right abdominal pain. Guarding and rebound tenderness were denied. On initial physical examination, the blood pressure was 100/64 $\mathrm{mmHg}$ and the heart rate was 109 beats per min. Serum biochemistry showed a white blood cell count of $8.1 \times$ $10^{3} / \mu \mathrm{L}$, red blood cell count of $478 \times 10^{4} / \mu \mathrm{L}$, and hemoglobin $14.2 \mathrm{~g} / \mathrm{dL}$. CT showed a $72 \times 68 \mathrm{~mm}$ metastatic liver tumor in segment VI. The tumor rim was enhanced, but the center was not, and thus considered to consist of necrotic tissue. The tumor contained a 9-mm pseudoaneurysm, and fluid collection was visible around hepatic edge (Fig. 1). An intrahepatic artery pseudoaneurysm within the metastatic liver tumor was diagnosed, with the surrounding fluid indicating potential, active bleeding. An emergency angiography confirmed the presence of a pseudoaneurysm stemming from a branch of the hepatic artery in segment VI (Fig. 2). The pseudoaneurysm was cannulated and successfully embolized using microcoils. After embolization, there were no clinical signs, and the patient was discharged 9 days after the angiography without any recurrence of bleeding. Follow-up CT 1 month after the angiography detected enlarged liver and bone metastasis. In accordance with the wishes of the patient and his family, palliative care was begun. The patient remains alive with palliative care 5 months after the angiography.

\section{Discussion}

In this rare case, ITPA within a liver metastasis of gastric cancer following chemotherapy without any prior hepatobiliary intervention was ruptured and treated using arterial embolization.

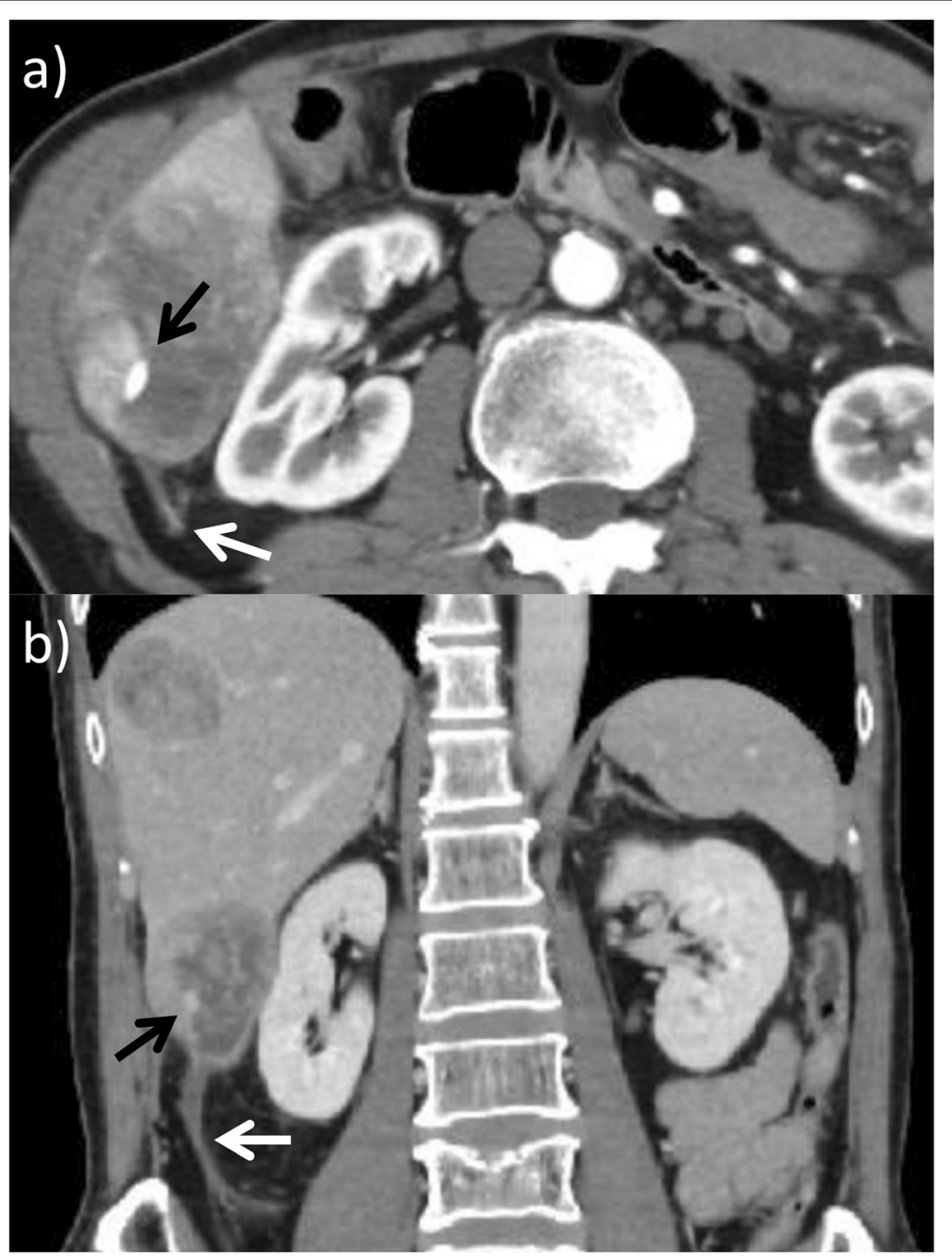

Fig. 1 a, b CT revealed a $72 \times 68 \mathrm{~mm}$ metastatic liver tumor in segment VI containing a 9-mm pseudoaneurysm (black arrow) and fluid collection around the hepatic edge (white arrow) 


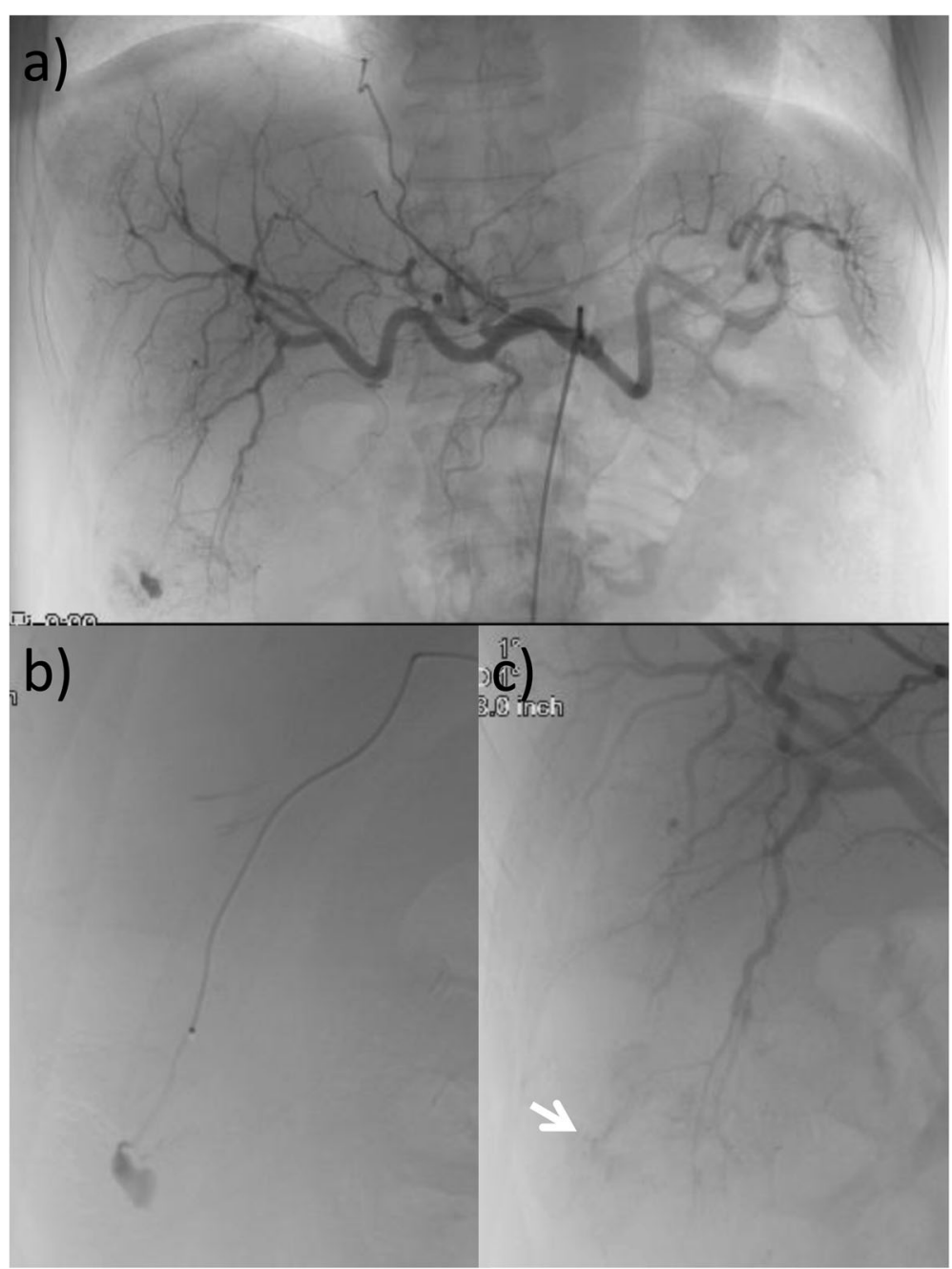

Fig. 2 a, b Emergency angiography showed a pseudoaneurysm stemming from a branch of the hepatic artery in segment VI. c The pseudoaneurysm was cannulated and successfully embolized using microcoils (white arrow)

Visceral artery aneurysms are rare. Aneurysms of the hepatic artery, celiac axis, and branches, and splenic artery have a prevalence of $39 \%, 39 \%$, and $18 \%$, respectively [3]. Of cases of hepatic artery aneurysm, $77 \%$ are confined to the segment proximal to the liver, $20 \%$ have combined intra- and extraparenchymal involvement, and 3\% are localized exclusively within the liver [4]. The present case was a pseudoaneurysm arising exclusively from the intrahepatic artery (branch of the segment VI hepatic artery).

The causes of visceral artery pseudoaneurysms are trauma, infection, inflammatory diseases, and complications of abdominal surgery, hepatobiliary interventions, and endoscopic explorations [5]. On the other hand, the incidence of pseudoaneurysms caused by malignant tumors is low although pseudoaneurysms caused by hepatocellular carcinomas (HCC), malignant lymphomas, desmoids, neurofibromatosis, giant cell tumors, leukemia, and so on have been reported [6-13].
An ITPA arising from the intrahepatic artery within a liver tumor is rare. While some cases of ITPA in the liver have been reported, almost all these tumors were hepatocellular carcinomas (HCC) $[6-8,14,15]$. ITPA within a liver metastasis of gastric cancer is extremely rare (Table 1). Liu et al. reported such a case caused by vascular injury after radiofrequency ablation (RFA) [16].

Intrahepatic artery pseudoaneurysms are iatrogenic, resulting from hepatobiliary intervention [17]. Even in ITPA, hepatobiliary interventions like transcatheter arterial chemoembolization and RFA resulting in vascular catastrophe are the most common causes [14-16, 18]. Yoshikawa et al. reported a case of ITPA within an HCC after carbon ion radiotherapy and described the cause as being angiogenesis, fragmentation of the vascular mesothelial elastic fibers, and edema of the subcutaneous blood vessels due to radiation [8]. However, few researchers have reported pseudoaneurysms arising de 
Table 1 Clinical characteristics of patients with ITPA within a liver metastasis of gastric cancer

\begin{tabular}{llllllllll}
\hline Author & Gender & Age & Symptom & Prior intervention & Tumor size $(\mathrm{mm})$ & Site & ITPA size $(\mathrm{mm})$ & Treatment & Outcome \\
\hline Liu [16] & $\mathrm{M}$ & 77 & Hematemesis & RFA & 45 & Segment III & Not described & Embolization & Alive \\
Our case & $\mathrm{M}$ & 59 & Abdominal pain & none & 72 & Segment VI & 9 & Embolization & Alive
\end{tabular}

ITPA intratumoral pseudoaneurysm, RFA radiofrequency ablation

novo from within a HCC without any prior intervention $[6,7]$. Among these, Haider et al. reported a case series (including 25 cases) and an annual incidence of $0.24 \%$ for the condition. The development of pseudoaneurysms is thought to be related to tumor angiogenesis [7].

In the present case, the patient had no hypertension, cardiovascular history, inflammatory disease, or recent trauma, and the metastatic liver tumor had not been treated by any hepatobiliary or other surgical procedure or radiation. Anatomical abnormalities, such as vascular malformation and tumor angiogenesis, were not observed on either the CT or angiogram. The cause of the ITPA in the present case was unclear, but the tumor necrosis found on CT likely led to encasement, erosion of the vessel wall, and subsequent arterial wall weakening leading to the development of the pseudoaneurysm as the tumor progressed. The chemotherapeutic drugs administered to the patient may have contributed to the conditions favoring its development. Two cases of pseudoaneurysm developing after FOLFIRI (irinotecan, leucovorin, 5-fluorouracil: 5-FU) combined with bevacizmab and FOLFOX (oxaliplatin, leucovorin, 5-FU) have been reported $[19,20]$. Two other cases involved acute enlargement of an abdominal aortic aneurysm following chemotherapy with gemcitabine, cisplatin, docetaxel, and 5-FU [21, 22]. Some chemotherapy drugs have vascular toxicity and induce cell apoptosis leading to loss of integrity of the vascular wall [19-23]. In the present case, the ITPA was diagnosed during nivolumab administration as second-line chemotherapy following $\mathrm{S}-1$ as adjuvant chemotherapy and RAM plus PTX as first-line chemotherapy. A previous study reported that 5-FU, its oral pro-drug, and PTX primarily alter the molecular signaling pathways controlling vascular smooth muscle cell tone, thereby inducing vasoconstriction, but did not explain the relationship of these drugs to pseudoaneurysm formation [23]. RAM is a monoclonal antibody that binds to vascular endothelial growth factor (VEGF) receptor-2, preventing its activation. Bleeding is a major adverse event reported in some clinical trials caused by angiogenesis inhibitors disrupting the tumor vasculature by inhibiting VEGF signaling, leading to thrombosis or bleeding [24, 25]. RAM may inhibit endothelial growth, thus resulting in a vessel wall breach and pseudoaneurysm formation. However, due to the absence of reports of similar cases, it is unclear whether any correlation exists between RAM and ITPA formation. However, such a correlation cannot be entirely ruled out, and it is possible that RAM is responsible for the bleeding caused by an ITPA rupture.

The prevalence of hepatic artery pseudoaneurysm ruptures can be as high as 90\% [26]. As patients with visceral artery aneurysm rupture frequently present with hemorrhagic shock, prompt resuscitation with blood products and hemorrhage control are critical [27]. There are several effective approaches for treating hepatic artery pseudoaneurysms, including open surgery, which has a $21 \%$ mortality rate, as well as endovascular methods, which have a low complication and mortality rate [28]. Recent interventions using arterial embolization or stent grafts have been proposed as alternatives to surgical repair and offer real advantages in terms of survival [3].

\section{Conclusion}

We reported a case of intratumoral pseudoaneurysm within a liver metastasis of gastric cancer detected during chemotherapy. It is necessary to recognize that pseudoaneurysms can arise within a metastatic liver tumor during chemotherapy.

\section{Abbreviations \\ CT: Computed tomography; FOLFIRI: Irinotecan, leucovorin, 5-fluorouracil; FOLFOX: Oxaliplatin, leucovorin, 5-FU; HCC: Hepatocellular carcinoma; ITPA: Intratumoral pseudoaneurysm; PTX: Paclitaxel; RAM: Ramucirumab; RFA: Radiofrequency ablation; VEGF: Vascular endothelial growth factor}

\section{Acknowledgements}

None

Authors' contributions

$\mathrm{KI}$ performed the surgery. TK performed the endovascular procedure. $\mathrm{HO}$ and $\mathrm{YI}$ carried out the data acquisition and drafted the manuscript. SY, RY, $\mathrm{FH}, \mathrm{KI}$, and YM revised the article. The author(s) read and approved the final manuscript.

\section{Funding}

None

\section{Availability of data and materials}

The data are not available for public access due to patient privacy concerns but are available from the corresponding author on reasonable request.

Ethics approval and consent to participate

The present study was conducted in accordance with the ethical standards of our institution.

\section{Consent for publication}

Written informed consent was obtained from the patient for publication of this case report and any accompanying images.

Competing interests

The authors declare that they have no competing interests. 


\section{Author details}

'Department of Surgery, Tokyo Metropolitan Tama Medical Center, 2-8-29 Musashidai, Fuchu-shi, Tokyo 183-8524, Japan. ²Department of Surgery, Tokyo Metropolitan Matsuzawa Hospital, 2-1-1 Kamikitazawa, Setagaya-ku, Tokyo 156-0057, Japan. ${ }^{3}$ Department of Radiology, Tokyo Metropolitan Tama Medical Center, 2-8-29 Musashidai, Fuchu-shi, Tokyo 183-8524, Japan.

Received: 23 December 2019 Accepted: 12 February 2020

Published online: 18 February 2020

\section{References}

1. Núñez DB, Torres-León M, Múnera F. Vascular injuries of the neck and thoracic inlet: helical CT-angiographic correlation. RadioGraphics. 2004; 24:1087-98.

2. Sano T, Kodera Y. Japanese gastric cancer treatment guidelines 2010 (ver. 3). Gastric Cancer. 2011;14:113-23.

3. Tulsyan N, Kashyap VS, Greenberg RK, Sarac TP, Clair DG, Pierce G, et al. The endovascular management of visceral artery aneurysms and pseudoaneurysms. J Vasc Surg. 2007;45:276-83.

4. Berceli SA. Hepatic and splenic artery aneurysms. Semin Vasc Surg 2005;18:196-201.

5. Boufi M, Belmir H, Hartung O, Ramis O, Beyer L, Alimi YS. Emergency stent graft implantation for ruptured visceral artery pseudoaneurysm. J Vasc Surg. 2011:53:1625-31

6. Chingkoe CM, Chang SD, Legiehn GM, Weiss A. Hepatic artery pseudoaneurysms arising from within a hepatocellular carcinoma. $\mathrm{Br} \mathrm{J}$ Radiol. 2010;83:252-4.

7. Haider Z, Idris M, Sajjad Z, Humayun S, Kashif N, Ali S. Intratumoral pseudoaneurysms in hepatocellular carcinoma: do they occur de novo without any prior intervention? A tertiary care center experience of 6 years. Acta radiol. 2015:56:1027-33.

8. Yoshikawa S, Asano T, Watanabe M, Ishii T, Ohtake H, Fujiwara J, et al. Rupture of hepatic pseudoaneurysm formed nine years after carbon ion radiotherapy for hepatocellular carcinoma. Intern Med. 2019;58:2639-43.

9. Hiraoka T, Komiya T, Tsuneyoshi H, Shimamoto T. Thoracic pseudoaneurysm caused by malignant lymphoma. Ann Thorac Surg. 2018;105:e19-20.

10. Blanes Ortí PC, Bernal LR, Requejo García L, Hernández MM. Abdominal aortic rupture secondary to lymphoma recurrence. Ann Vasc Surg. 2019; 58:381.e5-9.

11. Smith BL, Munschauer CE, Diamond N, Rivera F. Ruptured internal carotid aneurysm resulting from neurofibromatosis: treatment with intraluminal stent graft. J Vasc Surg. 2000;32:824-8.

12. Fraile NMP, Toloi D, Kurimori CO, Matutino ARB, Codima A, Camargo VP, et al. Case report successful intravascular correction of intratumoral pseudoaneurysm by erosion of the aorta in a patient with thoracic giant cell tumor of bone responding to denosumab. Case Rep Oncol Med. 2015; 2015:626741.

13. Kim MD, Kim H, Kang SW, Jeong BG. Nontraumatic hepatic artery pseudoaneurysm associated with acute leukemia: a possible complication of pyogenic liver abscess. Abdom Imaging. 2002;27:458-60.

14. Tamai F, Furuse J, Maru Y, Yoshino M. Intrahepatic pseudoaneurysm: a complication following radio-frequency ablation therapy for hepatocellular carcinoma. Eur J Radiol. 2002;44:40-3.

15. Chuang $\mathrm{CH}$, Chen $\mathrm{CY}$, Tsai HM. Hepatic infarction and hepatic artery pseudoaneurysm with peritoneal bleeding after radiofrequency ablation for hepatoma. Clin Gastroenterol Hepatol. 2005;3:A23.

16. Liu CA, Chiu NC, Chiou YY. Massive hematemesis after radiofrequency ablation of metastatic liver tumor with successful hemostasis achieved through transarterial embolization. Clin Imaging. 2018:51:192-5.

17. Priyadarshi RN, Kumar R, Anand U. Case report: spontaneous resolution of intracavitary hepatic artery pseudoaneurysm caused by amebic liver abscess following percutaneous drainage. Am J Trop Med Hyg. 2019;101:157-9.

18. Evangelos T, Vassiliki $P$, Dimitrios $K$, Theodoros $P$. Hepatic artery aneurysm complicating intra-arterial chemotherapy for hepatocellular carcinoma. Hepatogastroenterology. 2003;50:830-1.

19. Cosic L, Theivendren M, Spanger M, Weinberg L. Popliteal pseudoaneurysm after FOLFOX chemotherapy for metastatic colorectal cancer. Int I Surg Case Rep. 2019:63:1-4

20. Li C, Tsai H, Huang C, Yeh Y, Tsai T, Wang J. latrogenic pseudoaneurysm after bevacizumab therapy in patients with metastatic colorectal cancer: two case reports. Mol Clin Oncol. 2018:499-503.
21. Zanow J, Leistner Y, Ludewig S, Rauchfuss F, Settmacher U. Unusual course of an abdominal aortic aneurysm in a patient treated with chemotherapy for gastric cancer. J Vasc Surg. 2012;55:841-3.

22. Palm SJ, Russwurm GP, Chang D, Rozenblit AM, Ohki T, Veith FJ. Acute enlargement and subsequent rupture of an abdominal aortic aneurysm in a patient receiving chemotherapy for pancreatic carcinoma. J Vasc Surg. 2000; 32:197-200.

23. Herrmann J, Yang EH, Iliescu CA, Cilingiroglu M, Charitakis K, Hakeem A, et al. Vascular toxicities of cancer therapies: the old and the new - an evolving avenue. Circulation. 2016;133:1272-89.

24. Xiao B, Wang W, Zhang D. Risk of bleeding associated with antiangiogenic monoclonal antibodies bevacizumab and ramucirumab: a meta-analysis of 85 randomized controlled trials. Onco Targets Ther. 2018;11:5059-74.

25. Arnold D, Fuchs CS, Tabernero J, Ohtsu A, Zhu AX, Garon EB, et al. Metaanalysis of individual patient safety data from six randomized, placebocontrolled trials with the antiangiogenic VEGFR2-binding monoclonal antibody ramucirumab. Ann Oncol. 2017;28:2932-42.

26. O'Driscoll D, Olliff SP, Olliff JF. Hepatic artery aneurysm. Br J Radiol. 1999:72:1018-25.

27. Reiter DA, Fischman AM, Shy BD. Hepatic artery pseudoaneurysm rupture: a case report and review of the literature. J Emerg Med. Elsevier Ltd. 2013:44:100-3

28. Bashiri K, Roushan N, Hamidian SM. Hepatic artery pseudoaneurysm; simple or difficult to diagnose? Arch Iran Med. 2016:19:521-2.

\section{Publisher's Note}

Springer Nature remains neutral with regard to jurisdictional claims in published maps and institutional affiliations.

\section{Submit your manuscript to a SpringerOpen ${ }^{\circ}$ journal and benefit from:}

- Convenient online submission

- Rigorous peer review

- Open access: articles freely available online

- High visibility within the field

- Retaining the copyright to your article

Submit your next manuscript at $>$ springeropen.com 